\title{
Low-temperature thermodynamics of Xe-doped fullerite $\mathrm{C}_{60}$
}

\author{
M.S. Klochko and M.A. Strzhemechny \\ B. Verkin Institute for Low Temperature Physics and Engineering, of the National Academy of Sciences of Ukraine \\ 47 Lenin Ave., Kharkov 61103, Ukraine \\ E-mail: klochko@ ilt.kharkov.ua
}

Received December 12, 2014, published online April 23, 2015

\begin{abstract}
Using a model of the fullerene $\mathrm{C}_{60}$ molecule with carbon atoms uniformly distributed over its surface, the potential energy $U(\mathbf{n})$ of a Xe atom in an octahedral void of $\mathrm{C}_{60}$ is calculated. Within the framework of threedimensional harmonic oscillator, the lowest energy levels are estimated and the contribution of xenon impurity atoms to the heat capacity of the $\mathrm{Xe}-\mathrm{C}_{60}$ system is determined. The contribution of Xe dopants to the total heat capacity is shown to be essential compared to that of pure fullerite. Using the calculated energy spectrum we estimated the contribution of Xe atoms to the thermal expansivity of $\mathrm{C}_{60}$ with $37 \%$ of Xe. This contribution is in a qualitative agreement with experimental findings. We estimated the Grüneisen parameter $\Gamma$ due to the anisotropic part of $U(\mathbf{n})$ to show that the negative part of $\Gamma$ is negligible due to the very small width of the five lower oscillatory wave functions.
\end{abstract}

PACS: 61.48.+c Fullerenes and fullerene-related materials;

61.72.Ji Point defects (vacancies, interstitials; color centers, etc.) and defect clusters;

65.40.De Thermal expansion; thermomechanical effects;

65.40.Ba Heat capacity.

Keywords: doped fullerite $\mathrm{C}_{60}$, low-temperature thermodynamics, rare gases.

\section{Introduction}

The thermal expansivities $\alpha$ measured [1] in doped $\mathrm{C}_{60}$ were negative within certain temperature intervals; the authors related this effect with tunnel rotations of $\mathrm{C}_{60}$ molecules between the neighboring equilibrium states. However, even if negative warming was not documented, dopant-related expansivity as a function of temperature showed maxima [2]. Dolbin et al. explained it as being due to polyamorphic first-order transformations in orientational glasses, also related with tunnelling motion of $\mathrm{C}_{60}$ molecules. Both these interpretations were put to doubt, mostly because, first, $\mathrm{C}_{60}$ has a huge rotational moment and, second, the corresponding rotational barriers are very high and steep, so the maxima observed on the $\alpha(T)$ curves could be easily explained as being due to impurities, especially, if they are molecules [3].

However, even in the case of an atomic (RG) impurity such effect is still possible. In particular, it was shown [4] that the excited states of a Xe atom in octahedral voids can be represented as an oscillatory rotational modes. The resulting spectrum resembled, with slight modifications, the Devonshire spectra for molecular dopant species, that is, the thermodynamic quantities of $\mathrm{C}_{60}$ with rare gas impurities in octahedral voids could include negative contribu- tions to the respective Grüneisen parameter $\Gamma$. Such contribution is usually associated with energy spectra specifically modified due to some tunnelling motion, which in the case of rare gas atomic impurities were due to tunnelling in the angular space.

Since rather detailed $\mathrm{x}$-ray data are available [5] for the same $\mathrm{C}_{60}$-Xe sample that was used in thermal expansion measurements, it was evident that detailed thermodynamic calculations are needed to have better insight into the lowtemperature thermodynamics of this system.

\section{Interaction potential of Xe atom in O-void}

Let us consider a Xe atom in an octahedral cavity. The interaction between the Xe atom and any of the carbons atom that constitute the cage of six fullerene molecules $\mathrm{C}_{60}$ (see Fig. 1) will be described by the Lennard-Jones (LJ) potential with the parameters $\sigma=3.36 \AA$ and $\varepsilon=104 \mathrm{~K}$, as reconstructed from rare gas scattering on graphite surfaces [6]. Any of the six $\mathrm{C}_{60}$ molecules will be represented by a sphere of radius [7] $r_{0}=3.54 \AA$ with carbon atoms distributed homogeneously over the surface of every fullerene sphere with the density $60 /\left(4 \pi r_{0}^{2}\right)$.

Integration over the surfaces of the six nearest fullerene molecules (the contribution of the farther molecules is ig- 


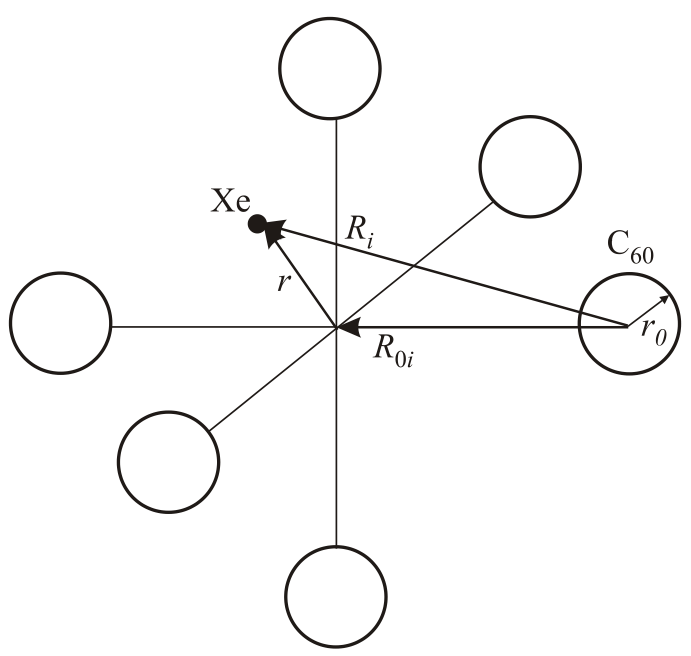

Fig. 1. Geometry of the problem, showing an octahedral cage with a xenon atom shifted from the void center.

nored) yields the following expression for the potential energy of a Xe atom in the octahedral void

$$
\begin{aligned}
\Phi(\mathbf{r}) & =\sum_{i=6}^{6} \frac{12 \varepsilon \sigma^{12}}{\left|\mathbf{R}_{0 i}+\mathbf{r}\right| r_{0}}\left\{R_{i-}^{-10}-R_{i+}^{-10}\right\}- \\
& -\sum_{i=6}^{6} \frac{30 \varepsilon \sigma^{6}}{\left|\mathbf{R}_{0 i}+\mathbf{r}\right| r_{0}}\left\{R_{i-}^{-4}-R_{i+}^{-4}\right\}
\end{aligned}
$$

where $R_{i-}=\left(\left|\mathbf{R}_{0 i}+\mathbf{r}\right|-r_{0}\right)$ and $R_{i+}=\left(\left|\mathbf{R}_{0 i}+\mathbf{r}\right|+r_{0}\right) ; \mathbf{r}$ is the radius-vector of the integration point; $\mathbf{R}_{0 i}$ is the radius-vector of the ith $\mathrm{C}_{60}$ molecule. Further analytical calculations are impractical, so we need to make certain approximation when dealing with the numeric sums in Eq. (1).

Since the problem has a cubic (octahedral) symmetry, we can represent the energy $\Phi(\mathbf{r})$ in the form

$$
\Phi(\mathbf{r})=\sum_{\text {even } N} U_{N}(r) I_{N}(\mathbf{n})
$$

where $\mathbf{r}=r \mathbf{n}, \quad I_{N}(\mathbf{n})$ are the cubic invariants of rank $N$. Here we also remind that $I_{2}(\mathbf{n})=0$.

To seek the solution to the respective Schrödinger equation, as the first step we truncate the potential energy of Eq. (2), leaving only two terms with $N=0$ and $N=4$, which are proportional to the respective invariants

$$
\begin{gathered}
I_{0}(\mathbf{n})=1 / \sqrt{4 \pi} \\
I_{4}(\mathbf{n})=\sqrt{\frac{7}{12}} Y_{40}(\mathbf{n})+\sqrt{\frac{5}{24}}\left(Y_{44}(\mathbf{n})+Y_{4 \overline{4}}(\mathbf{n})\right) .
\end{gathered}
$$

The respective energy functions $U_{N}(r)$ can be calculated numerically:

$$
U_{N}(r)=\int \Phi(r \mathbf{n}) I_{N}(\mathbf{n}) d \mathbf{n} .
$$

As the second step we calculate $U_{0}(r)$ :

$$
U_{0}(r)=\int \frac{\Phi(r \mathbf{n})}{\sqrt{4 \pi}} d \mathbf{n},
$$

for the value $a_{0}=14.04 \AA$, which is the $T=0$ value in pure $\mathrm{C}_{60}$. This is a preliminary calculation in view of the next steps explained below.

The third step consists in complying our estimates with the structure data [5] on a $37 \%$ Xe-doped $C_{60}$ sample. First we need to know how the depth of $U_{0}(R)$ depends on $R_{0}=a / 2$. As it follows from the $U_{0}(R)$ curves calculated as shown in Eq. (6) for different values of $R_{0}$ (see also Fig. 2), the lowest value of the minimum is at $R_{0}^{(\min )} \simeq 6.98 \AA$. This means that the octahedral void would tend to shrink to values less even than the value of $R_{0}^{(0)}=7.02 \AA$ in pure $\mathrm{C}_{60}$, whereas in the above-mentioned doped sample $R_{0}^{(\exp )}=7.035 \AA$. By increasing $\sigma$ we were able to shift $R_{0}^{(\min )}$ to higher values until it surpassed $R_{0}^{(\exp )}$. Then we took into consideration the fact that $\mathrm{C}_{60}$ resists elastically above (or constriction below) the value $R_{0}^{(0)}$ in pure $\mathrm{C}_{60}$ at zero temperature. The latter value is determined exclusively by the interactions of $\mathrm{C}_{60}$ molecules between themselves. The corresponding energy per octahedral site can be easily derived [13] to be

$$
U_{\mathrm{el}}\left(R_{0}\right)=9 K r\left[R_{0}-R_{0}^{(0)}\right]^{2} .
$$

Here $K$ is the low-temperature bulk modulus known from experiment [8,9] and $R_{0}$ (which depends on $\sigma$ ) is the distance from the center of octa-void to the $\mathrm{C}_{60}$ molecule which belongs to the octahedral cage.

For every given value of $\sigma$ in a subsequent iteration procedure we evaluated the resulting value of $R_{0}$ with account of the fact that the $\mathrm{C}_{60}$ lattice elastically resists extension (or shrinking). This was done by solving the following equilibrium equation

$$
\frac{d}{d R_{0}}\left[U_{0}\left(R_{0}, \sigma\right)+U_{\mathrm{el}}\left(R_{0}\right)\right]=0 .
$$

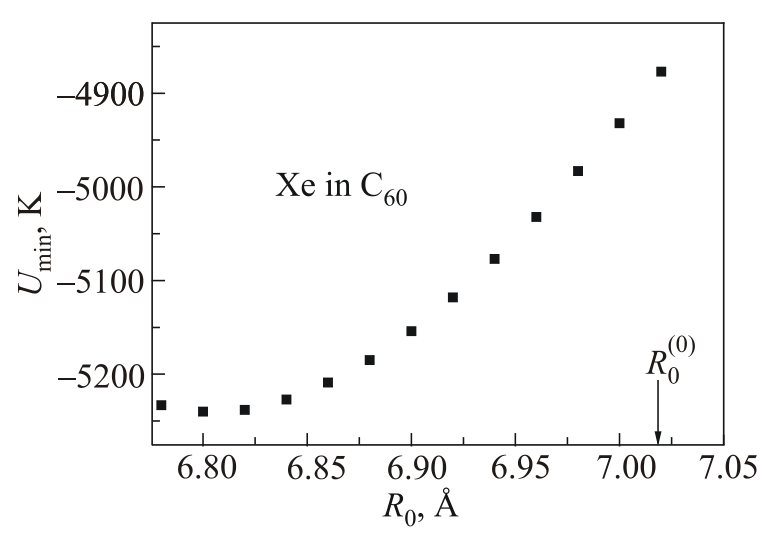

Fig. 2. The potential well depth of $U_{0}^{(\mathrm{final})}(r)$ as a function of the half of the lattice parameter $a$ of fullerite $\mathrm{C}_{60}, R_{0}=a / 2$. 
If the solution $R_{0}^{\text {fin }}$ was not equal to $R_{0}^{(\exp )}=7.035 \AA$, we varied $\sigma$ until it was. In this way we determined the optimum value of $\sigma$ which was used to calculate the $\Phi(r)$ as in Eq. (1) and then, using Eq. (6), the final potential curve $U_{0}^{(f i n)}(R)$ to be utilized in further estimations.

We first perform the final fitting (cf. Fig. 3) of $U_{0}^{(\text {final) }}(R)$ to a $R^{2}$ function within the range $0 \leq R \leq 0.25 \AA$ and obtain the eigenvalue of the oscillation energy $\hbar \omega / k=$ $=(64.93 \pm 0.45) \mathrm{K}$, where $k$ is the Boltzmann constant.

For a 3D oscillator the eigenvalues for the $n$th excited state are $E_{n}=\hbar \omega(n+3 / 2)$ and degeneracies $g_{n}=$ $=(1 / 2)(n+1)(n+2)$. Since we are going to take into account the five lowest oscillatory states, in Fig. 4 we show the modulus of the eigenfunction with $n=4$ (the explicit expressions for eigenfunctions can be found elsewhere $[4,10])$; the ground-state wave function is roughly twice as narrow. The span of this eigenfunction justifies our choice for the width of the fitting range for the evaluation of $\omega$.

One of our goals is to understand to what extent the rotational tunnelling levels are split by the $N=4$ potential term (cf. Eqs. (2) and (5)). To that end, proceeding from the general considerations that for sufficiently smooth functions involved, as the actual case is, the small-argument asymptote of $U_{N}(x)$ must be $x^{N}$, we obtained that $U_{4}(R) \simeq B R^{4}$ with $B=(7529 \pm 33) \mathrm{K}^{-4}$. In our subsequent numeric estimations we will use the following dependence:

$$
U_{4}(R) / \hbar \omega=\gamma(R / \rho)^{4}
$$

where $\rho=(\hbar / M \omega)^{1 / 2}=0.106 \AA$ is the natural reference distance in the quantum problem of harmonic oscillator and $\gamma \simeq 0.015$ is the dimensionless parameter which determines the splitting of the energy levels due to the anisotropic potential $U_{4}(R, \mathbf{n})$.

Now we evaluate the changes in the excitation spectrum as well as the effects in low-temperature thermal expansion due to angular tunnelling. From scratch we note that the

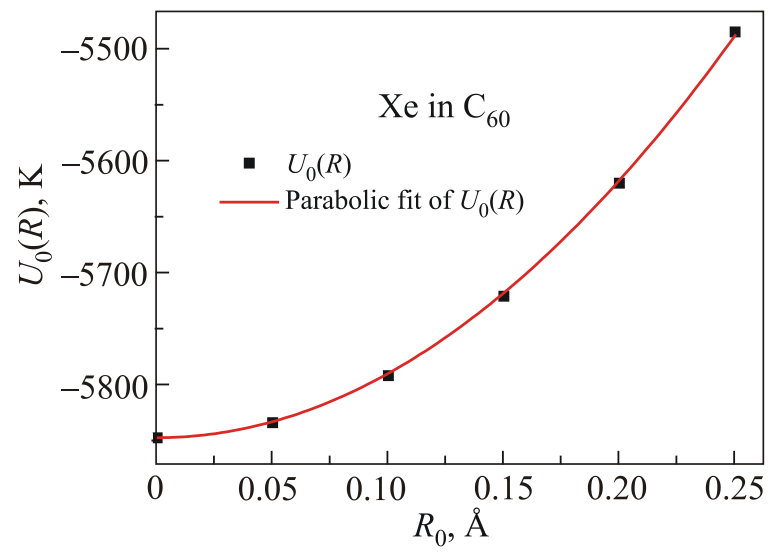

Fig. 3. Potential energy of a Xe atom inside an octahedral cage as a function of the shift from cage center.

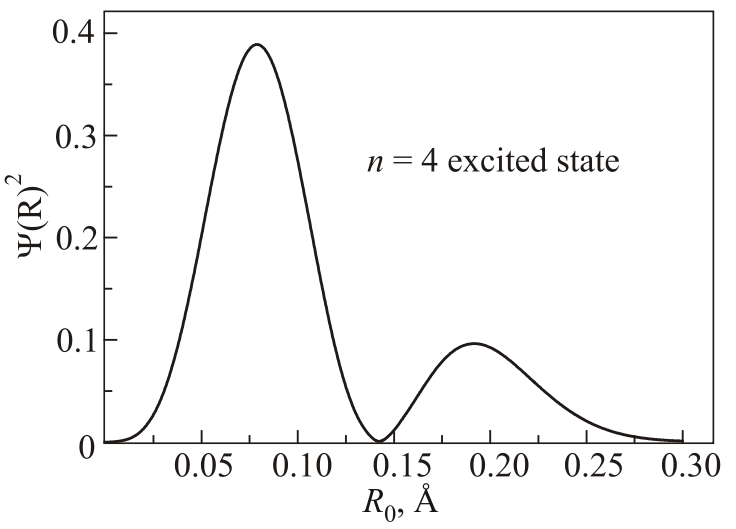

Fig. 4. The shape of the wave function of the $n=4$ excited state.

total span of the wave functions of the lowest five levels do not exceed $0.25 \AA$. That, in its turn, means that at these distances the contribution of the correction term $U_{4}(r) I_{4}(\mathbf{n})$ must be inessential. Since the eigen-functions of the $3 \mathrm{D}$ oscillator are well known $[4,10]$, it is easy to evaluate the corresponding splittings. Thus we found that the splitting of, for example, the fourth excited level amounts to $1.4 \mathrm{~K}$. That means that the respective negative contribution for the low-temperature thermal expansion of Xe-doped $\mathrm{C}_{60}$ is very small compared to the classical contribution. Comparing with a similar contribution due to a molecular dopant, for instance, a nitrogen molecule with its bond length (ana$\log$ of diameter) of $1.1 \AA$, it is understandable that its radius of $0.55 \AA$ is sufficient to probe ranges closer to the octahedral cage molecules, rendering the splitting much larger than in our case of the atomic Xe dopant.

\section{Heat capacity}

Now we calculate the Xe-related contribution to the lowtemperature heat capacity of the above-mentioned sample with $37 \%$ of the Xe occupancy. To this end we use the following expression for the free energy per dopant particle

$$
F=-k T \ln \sum_{n} g_{n} \exp \left(-E_{n} / k T\right),
$$

where $g_{n}$ is the degeneracy of level $n$. In Fig. 5 we show the experimental data [11] for pure $\mathrm{C}_{60}$ (solid triangles) as well three theoretical curves as explained in the caption. We should note that only the five lower energy levels were taken into account in our estimates. The particular fraction of $37 \%$ in octahedral voids was taken, because this fraction was in a real sample in which the thermal expansion was measured at low temperatures [8] (see also the next Section). In Fig. 5 one can see that presence of xenon atoms even at a level of $37 \%$ changes the $C_{V}(T)$ dependence significantly compared with that for pure $\mathrm{C}_{60}$. If all octahedral voids were filled, there would have been a maximum at $T \simeq 15 \mathrm{~K}$. 


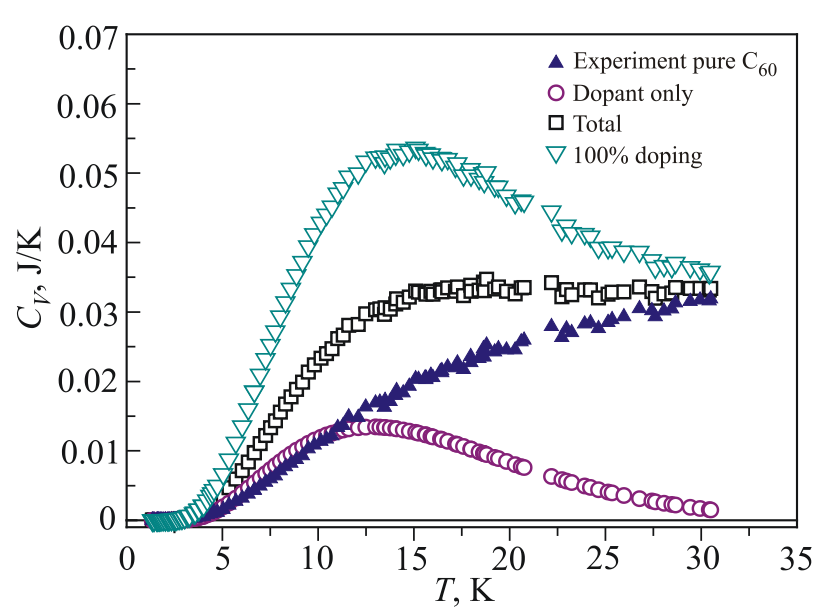

Fig. 5. Heat capacity of pure and Xe-doped $\mathrm{C}_{60}$ : solid triangles show experimental data [11] for pure fullerite; theory: contribution of $37 \%$ of Xe in octahedral voids (empty circles); a sum of $C_{V}$ of pure $\mathrm{C}_{60}$ and the contribution of $37 \%$ of Xe (empty squares); a sum of $C_{V}$ of pure $\mathrm{C}_{60}$ and the contribution of $100 \%$ of Xe (empty overturned triangles).

\section{Thermal expansion}

Evaluation of the low-temperature linear thermal expansion coefficient $\alpha$ as a function of temperature was carried out with the aid of the known relation [12]

$$
\left.\alpha(T)=\left(\chi_{T} / 3 V k T^{2}\right)\left\{\left\langle E_{i}^{2} \Gamma_{i}\right\rangle-\left\langle E_{i}\right\rangle\right\rangle E_{i} \gamma_{i}\right\rangle .
$$

Here the thermodynamic average is defined as

$$
\left\langle x_{i}\right\rangle=\frac{\sum_{i} f_{i} x_{i} \exp \left(-E_{i} / k T\right)}{\left.\sum_{i} f_{i} \exp \left(-E_{i} / k T\right)\right\rangle}
$$

where $\chi_{T}$ is the isothermal compressibility and

$$
\Gamma_{i}=-d \ln E_{i} / d \ln V
$$

is the partial Grüneisen parameter.

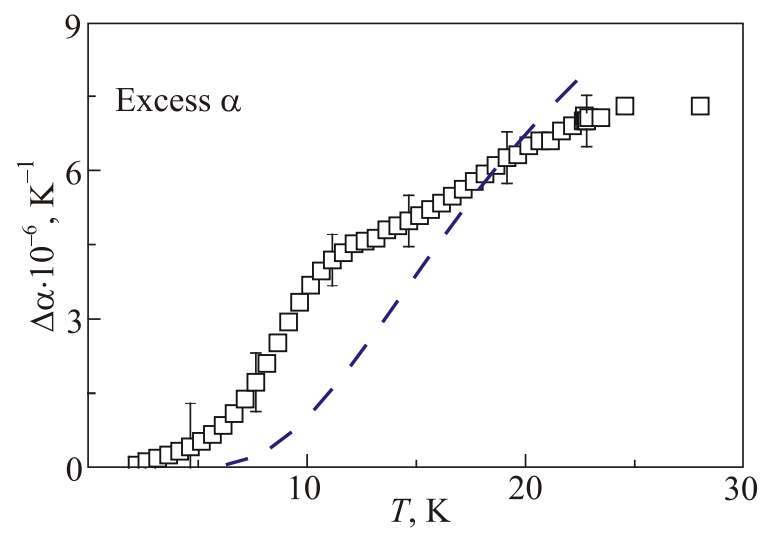

Fig. 6. Linear thermal expansivity of Xe-doped $\mathrm{C}_{60}$ : experiment [1] (empty squares) and theory (dashed curve).
As we noted above, the tunnelling-related contribution to $\Gamma$ is negligible, the remaining $\Gamma$ associated with the Xe impurities will be the same for all oscillatory levels, because the energy spacings between oscillatory levels of Xe atoms depend on the volume of octahedral void but are the same for all levels. We calculate the respective positive $\Gamma$ to be 6.89. In Fig. 6 we compare our estimates of the linear expansivities as a function of temperature with the experimental data.

\section{Conclusions}

In order to analyze the available experimental data concerning the thermodynamic properties (heat capacity and thermal expansivity) of $\mathrm{C}_{60}$ doped (partially) with xenon, we calculated the potential energy $U(\mathbf{R})$ of a xenon atom in an octahedral void of $\mathrm{C}_{60}$ making use of the LJ potential. The relevant LJ parameters used were close to those which were derived from scattering of Ar atoms from graphite surfaces [6], the range parameter $\sigma$ being varied to fit the actual lattice parameter [5] of fullerite $\mathrm{C}_{60}$ doped with $37 \%$ Xe.

We calculated the temperature dependence of the contribution due to the $37 \%$ Xe doping to the low-temperature heat capacity of $\mathrm{C}_{60}$ and showed that this contribution is quite appreciable and would change the net result. Unfortunately, heat capacities of Xe-doped samples were not measured.

We have also evaluated the contribution of $37 \% \mathrm{Xe}$ to the linear low-temperature thermal expansion $\alpha(T)$ and compared it with the corresponding contribution measured [1] on such a sample. Again, like $C(T), \alpha(T)$ has a characteristic low and broad maximum close to $28 \mathrm{~K}$. Our calculations are in a reasonably good agreement with the available experimental data, without resorting to an assumption of tunnelling rotations of $\mathrm{C}_{60}$ molecules or phase transitions between different realizations of the actual orientational glass state.

We evaluated the splittings of the lowest four excited vibrational level of a xenon atom in an octahedral cavity of $\mathrm{C}_{60}$ lattice owing to the anisotropic part of the potential energy of Xe in octahedral void. It was shown that the splittings are small (about $0.14 \mathrm{~K}$ for the $n=4$ excited oscillatory level of the Xe atom). This result is understandable, considering that the wave functions even of higher excited states are spread to $0.3-0.4 \AA$ where the contribution of the anisotropic part of the potential $U(\mathbf{R})$ is negligible compared with its isotropic part.

The authors thank M.I. Bagatskii and V.V. Sumarokov for providing original heat capacity data for pure fullerite $\mathrm{C}_{60}$. Similar thanks are due to A.V. Dolbin for the detailed experimental data concerning thermal expansivities of both pure and Xe-doped fullerite $\mathrm{C}_{60}$. 
1. A.N. Aleksandrovskii, A.S. Bakai, D. Cassidy, A.V. Dolbin, V.B. Esel'son, G.E. Gadd, V.G. Gavrilko, V.G. Manzhelii, S. Moricca, and B. Sundqvist, Fiz. Nizk. Temp. 31, 565 (2005) [Low Temp. Phys. 31, 429 (2005)].

2. A.V. Dolbin, N.A. Vinnikov, V.G. Gavrilko, V.B. Esel'son, V.G. Manzhelii, G.E. Gadd, S. Moricca, D. Cassidy, and B. Sundqvist, Fiz. Nizk. Temp. 35, 299 (2009) [Low Temp. Phys. 35, 226 (2009)].

3. A.F. Devonshire, Proc. Roy. Soc. (London) A 153, 601 (1936).

4. M.A. Strzhemechny and I.V. Legchenkova, Fiz. Nizk. Temp. 36, 470 (2010) [Low Temp. Phys. 36, 370 (2010)].

5. A.I. Prokhvatilov, N.N. Galtsov, I.V. Legchenkova, M.A. Strzhemechny, D. Cassidy, G.E. Gadd, S. Moricca, B. Sundqvist, and N.A. Aksenova, Fiz. Nizk. Temp. 31, 585 (2005) [Low Temp. Phys. 31, 445 (2005)].

6. G. Vidali and M.W. Cole, Phys. Rev. B 29, 6736 (1984).
7. J.D. Axe, P.C. Chow, S.C. Moss, and P. Wochner, Physica B 219-220, 121 (1996).

8. S. Hoen, N.G. Chopra, X.-D. Xiang, R. Mostovoy, Jianguo Hou, W.A Vareka, and A. Zettl, Phys. Rev. B 46, 12737 (1992).

9. N.P Kobelev, R.K. Nikolaev, N.S. Sidorov, and Ya.M. Soifer, Fiz. Tverd. Tela 43, 2244 (2001) [Phys. Solid State 43, 2344 (2001)].

10. M. Moshinsky, The Harmonic Oscillator in Modern Physics: From Atoms to Quarks, Gordon and Breach (1969).

11. M.I. Bagatskii, V.V. Sumarokov, and A.V. Dolbin, Fiz. Niz. Temp. 37, 535 (2011) [Low Temp. Phys. 37, 424 (2011)].

12. T.H.K. Barron, J.G. Collins, and G.K. White, Adv. Phys. 29, 609 (1980).

13. L.D. Landau and E.M. Lifshitz, Theory of Elasticity, Pergamon Press (1970). 